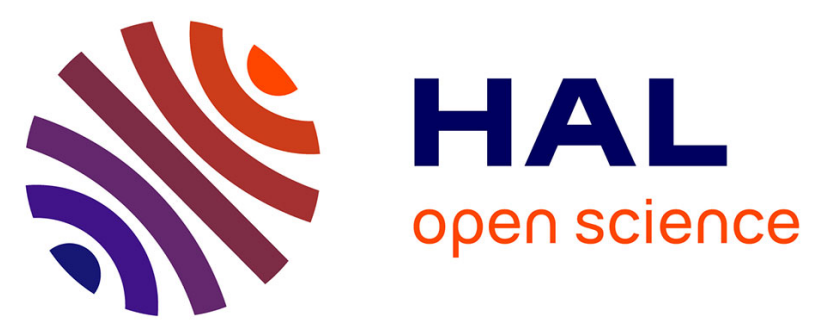

\title{
Collective fabrication of all-organic microcantilever chips based on a hierarchical combination of shadow-masking and wafer-bonding processing methods
}

Georges Dubourg, Ludivine Fadel-Taris, Claude Pellet, Isabelle Dufour, Cédric Ayela

\section{To cite this version:}

Georges Dubourg, Ludivine Fadel-Taris, Claude Pellet, Isabelle Dufour, Cédric Ayela. Collective fabrication of all-organic microcantilever chips based on a hierarchical combination of shadow-masking and wafer-bonding processing methods. Journal of Micromechanics and Microengineering, 2011, 21, 095021 (9 p.). 10.1088/0960-1317/21/9/095021 . hal-00609646

\section{HAL Id: hal-00609646 https://hal.science/hal-00609646}

Submitted on 13 Nov 2014

HAL is a multi-disciplinary open access archive for the deposit and dissemination of scientific research documents, whether they are published or not. The documents may come from teaching and research institutions in France or abroad, or from public or private research centers.
L'archive ouverte pluridisciplinaire HAL, est destinée au dépôt et à la diffusion de documents scientifiques de niveau recherche, publiés ou non, émanant des établissements d'enseignement et de recherche français ou étrangers, des laboratoires publics ou privés. 


\title{
Collective fabrication of all-organic microcantilever chips based on a hierarchical combination of shadow-masking and wafer- bonding processing methods
}

\author{
Georges Dubourg, Ludivine Fadel-Taris, Isabelle Dufour, Claude Pellet and Cédric Ayela \\ Université de Bordeaux, CNRS UMR 5218, IMS, 351 Cours de la Libération F-33405 Talence Cedex, \\ France.
}

\begin{abstract}
This paper describes a new collective microfabrication process of all-organic microcantilever chips. This method is based on the hierarchical combination of shadow-masking and wafer-bonding processes. The shadow-masking combines deposition and patterning in one step thanks to spray-coating through a polymer microstencil that gives the opportunity of patterning thermosensitive material such as PMMA. The shadow-masking parameters have been optimized to obtain suspended microcantilevers characterized by a convenient thickness profile. The resulting PMMA structures were then transferred onto SU-8 chips by using an SU-8 waferbonding process. The effect of the UV exposure dose of both SU-8 layers in contact on the bonding quality has been investigated and optimized. With the optimized bonding process we have achieved the large scale transfer of microstructures with a yield of $100 \%$ and a bond strength of 50 $\mathrm{MPa}$. These microcantilevers were also tested at resonance, to determine Young's moduli of patterned polymers. The low values obtained (below $5 \mathrm{GPa}$ ) make these organic MEMS structures strong candidates for highly sensitive sensing applications when used in the static mode.
\end{abstract}

\section{Introduction}

Silicon-based free-standing microcantilevers are widely used as micro- and nanoelectromechanical systems. More specifically, silicon cantilevers have shown great potential for sensing applications such as, molecular recognition $[1,2]$ or virus detection [3] in liquid media. These cantilevers generally operate in either the dynamic mode, where analytes binding on the cantilevers increase mass and thus decrease the resonant frequency, or in the static deflection mode, where analytes binding on one side of the cantilevers causes unbalanced surface stress resulting in a measurable mechanical deflection. In the dynamic mode, the operation of silicon microcantilevers in viscous fluids is limited by the fact that the quality factor ( $Q$ factor) is very low because of viscous damping and squeezing effects [4]. The static mode is usually preferred in liquid media but the deflection measurement may be difficult due to intrinsic rigidity of silicon, resulting in mechanical deflections that rarely exceed hundreds of nanometers. In this context, the introduction of polymers in microcantilever fabrication as an alternative solution to silicon is particularly promising. In fact, they are attractive for sensitive sensing applications in the static mode (bilayer effect) as single-use sensors due to their low cost, good processability, bio-compatibility [5] and tunable properties that may be achieved by an appropriate design of the materials [6]. Indeed, an organic free-standing structure is more flexible than a silicon one. Thus, the use of such low Young's modulus materials will enhance the transduction of molecular recognition using, for instance, an organic biomimetic sensitive layer, thus improving limits of detection of analytes. SU-8 epoxy negative photoresist is widely employed for organic microcantilever fabrication due to its high achievable aspect ratio, its chemical compatibility [7] and its versatility due to tunable properties obtained by mixing SU-8 with other materials. For instance, SU-8 may be made piezoresistive by mixing it with black carbon [8]. Generally, standard organic microcantilever fabrication methods are: (i) using a sacrificial layer, or (ii) sealing a free structure by transferring it to another layer (Figure 1) [9]. For (i), different polymers can be used as a sacrificial layer, including positive photoresists [10], metals [10] or thermally decomposable materials [11]. But this method is mostly limited by the chemical compatibility between the structural and sacrificial layers. For example, solvents used to remove the sacrificial layer [12] can also elute the structural one. Also, no concrete examples were found in the literature where thick films $(>50 \mu \mathrm{m})$ of positive photoresists, metals or thermally decomposable materials were used as a sacrificial layer. This is not desirable for organic microcantilevers where large deflections are expected in the static mode. For this, using uncrosslinked SU-8 as the sacrificial layer shows great potential. Indeed, it can maintain a flat 
Preprint - Journal of Micromechanics and Microengineering, vol. 21, 2011, 095021, 9 pages. (doi: 10.1088/0960-1317/21/9/095021).

surface (when crosslinked SU-8 is used for the supporting layer) for subsequent surface patterning of the structural layer, and can be etched selectively in the presence of different organic materials. However, at the moment, methods using uncrosslinked SU-8 as the sacrificial layer require a metallic UV-blocking coating between the structural and the sacrificial layers to protect this layer from UV radiation [13], or the use of complementary light absorption properties for the structural polymer [14]. Other reported fabrication methods that include the use of adhesive PET lamination to create three-dimensional flexible microfluidic networks [9]. Although the authors suggest that this technique could be used to fabricate suspended microstructures, no fabricated devices were reported. An alternative solution is to use the transfer method (ii) that can be combined with several processing methods such as nanoimprint lithography for the patterning of thermoplastics and biopolymers [9] or photolithography. However, a manual, one-by-one transfer of microstructures is most often observed in the literature $[15,16]$. Only two examples showed promising results for the wafer level fabrication of such organic devices. In a first approach, non-reticulated SU-8 was used to transfer SU-8 cantilevers, allowing an integrated readout of cantilevers behavior thanks to single-mode waveguides [17]. This process was then improved by using a partially reticulated SU-8 layer where the transferring layer could be patterned individually before bonding [18]. These pioneering works demonstrated the large scale fabrication of free-hanging organic microcantilevers. However, based on this approach, further improvements are necessary since: (i) a transparent wafer is mandatory for precise alignment of both substrates in contact during transfer, restricting the choice of substrates, (ii) qualitative characterization of bonding quality does not ensure a strong bond of transferred microstructures, with possible consequences on organic microcantilevers behavior, as already observed for resonant silicon ones $[19,20]$ and (iii) the fabrication process was validated only on SU-8 microcantilevers. Thus, the wafer level microfabrication of organic free-standing structures clamped on a substrate must be optimized to define a standard fabrication process.

In this context, we have combined two hierarchical processing methods to achieve the large-scale fabrication of all-organic microcantilever chips. First, the solution inspired from the wafer-level bonding of organic materials has been optimized for the rapid and collective microfabrication of organic freestanding cantilevers. Then, it is particularly interesting to combine this transfer method with a versatile patterning method where photosensitivity of materials is no longer mandatory for the structural layer. Indeed, some classes of organic materials such as biomaterials, gels, and thermoplastics are difficult or impossible to be structured with standard microfabrication methods based on optical lithography. To overcome this challenge, we have developed a shadow-masking approach dedicated to organic materials to create organic microcantilevers that remains compatible with other processing methods. Shadowmasking is a powerful method used for the micro- and nanopatterning of metals [21]. This method is based on the fabrication of a stencil where mechanical apertures allow the deposition and patterning of materials in one step. To our knowledge, only one example of the combination of shadow-masking with organic materials can be found in the literature for the fabrication of organic thin film transistors [22]. Thermal evaporation of an organic semiconductor was used for deposition, which is not compatible with higher molecular weight polymers, such as those used in the field of organic microsystems. In the present work, we propose a method where the organic structural material is spray-coated through a polymer microstencil, combining deposition and patterning in one step. This shadow masking approach does not require photo-masking of materials and allows patterning of most organic materials, i.e., photo- or thermosensitive ones, showing the great potential of this method in the field of organic microsystems. Moreover, using a flexible polymer microstencil, it can be easily adapted for the patterning on topographically rough, curved and unconventional surfaces $[23,24]$. Flexible stencils made of PDMS [24], parylene [25] or SU-8 [23] have shown great potential for metals patterning. Also, hybrid paryleneSU-8 microstencils have been used for the patterning of nontraditional materials [26]. In the present work, the microstencil is made of SU-8 photoresist and requires only two photolithography steps, making this solution consistent with the low-cost characteristic of organic MEMS. Also, SU-8 is a suitable material for microstencils fabrication since high aspect ratios can be obtained easily, combining high resolution with mechanical robustness. Thus, thanks to the hierarchical combination of shadow-masking and waferbonding processing methods, we have achieved the wafer level fabrication of PMMA suspended microcantilever chips characterized by a low Young's modulus and suitable for further integration in industrial processes, due to collective fabrication at low-cost. 
Preprint - Journal of Micromechanics and Microengineering, vol. 21, 2011, 095021, 9 pages. (doi: 10.1088/0960-1317/21/9/095021).

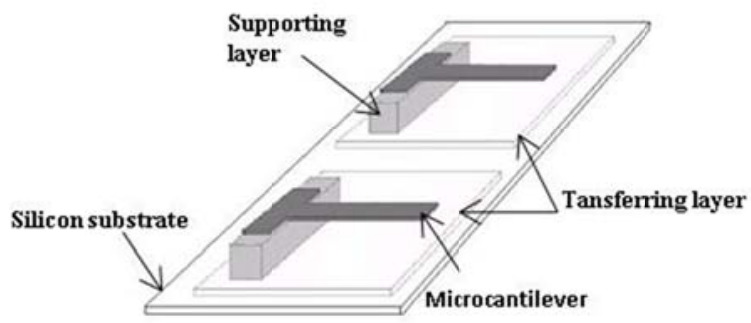

Figure 1. General scheme of organic free-standing microcantilevers obtained via the wafer-bonding processing method.

\section{Fabrication}

The process sequence for the fabrication of organic free-standing microstructures is schematically illustrated in Figure 2. The process uses an all-organic shadow-masking method combined with spraycoating to pattern the polymer structural layer on a temporary top wafer (Figure: 2.d). Then, this method has been combined with the wafer-bonding inspired technique to achieve the collective fabrication of free-standing microstructures. For this, SU-8 supports are introduced above the structural layer (Figure 2.g) while SU-8 transferring layers are patterned on a bottom wafer (Figure 2.f). The wafer bonding process results from compressing both wafers with a constant pressure and temperature (Figure 2.i). A mechanical self alignment of both wafers has been introduced by patterning pin and jig structures with an accuracy of $50 \mu \mathrm{m}$ (Figure 2.i). To complete the fabrication process, a sacrificial layer was initially coated onto the top wafer (Figure 2.c), allowing the release of microstructures after the transferring step by wet dissolution (Figure 2. j). The next sections detail the process sequences.

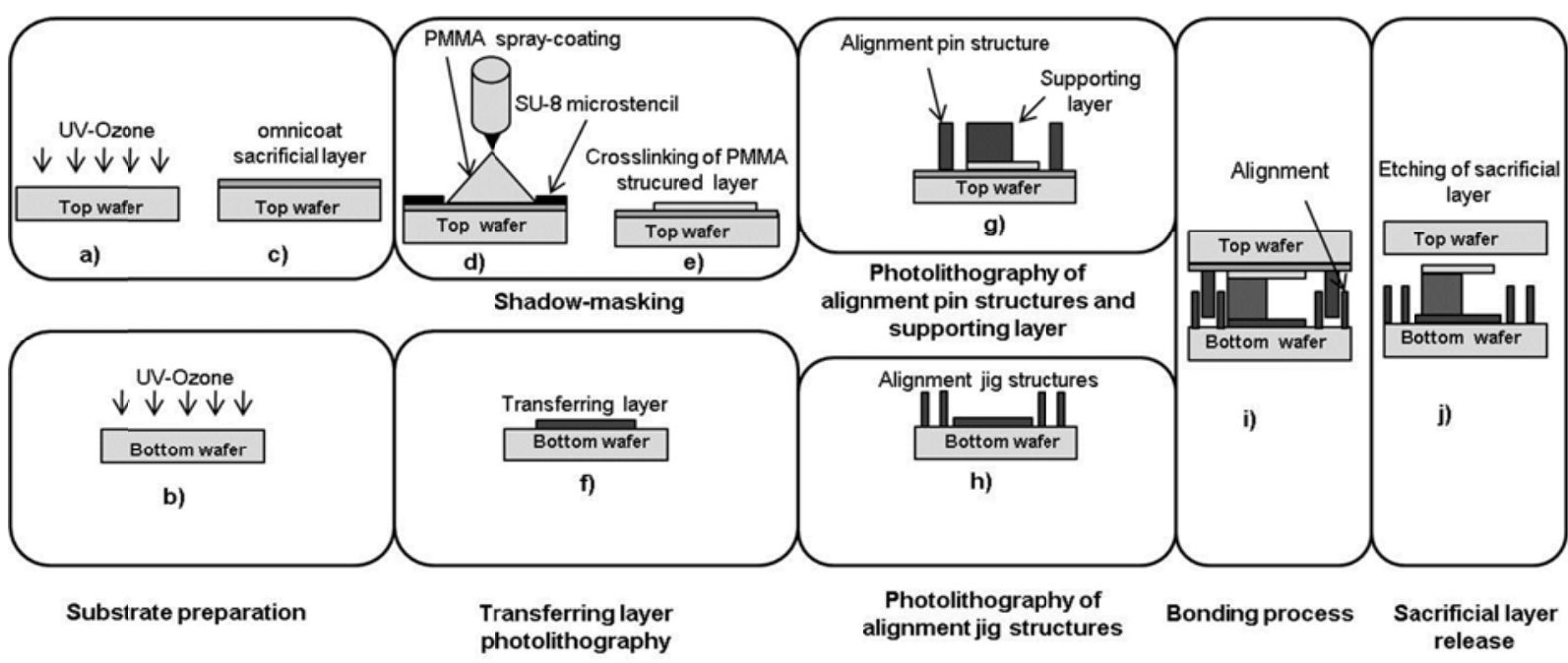

Figure 2. Fabrication process of organic microcantilevers: (a) and (b) Initial cleaning of top and bottom substrates (c) Deposition of omnicoat sacrificial layer $(200 \mathrm{~nm})$ on the top silicon substrate. (d) Patterning of PMMA microstructures by spray-coating through polymer microstencil. (e) Baking of PMMA at $180^{\circ} \mathrm{C}$ for 2 minutes. (f) Coating of the transferring layer onto the bottom wafer. (g) Patterning of SU-8 supporting layer and alignment pin structures characterized by a thickness of $100 \mu \mathrm{m}$. (h) Patterning of alignment jig structures. (i) Bonding of wafers with a constant pressure and temperature for $30 \mathrm{~min}$. (j) Removal of omnicoat layer for microstructures release

\subsection{Substrate preparation}

Prior to fabrication process, two silicon substrates must be prepared: a bottom substrate on which the 
Preprint - Journal of Micromechanics and Microengineering, vol. 21, 2011, 095021, 9 pages. (doi: 10.1088/0960-1317/21/9/095021).

structures will be clamped and a temporary top substrate used for structural layer patterning. Single side polished 4 inches silicon wafers with a thickness of $525 \mu \mathrm{m}$ were used as substrates. The two substrates were cleaned by using UV-Ozone for 10 minutes to improve adhesion of subsequent deposited layers (Figure 2.a and b). These substrates were rinsed with deionized water and dehydrated at $200{ }^{\circ} \mathrm{C}$ for $2 \mathrm{~h}$. On the top substrate, two thin layers of Omnicoat ${ }^{\mathrm{TM}}$ were successively spun at $1000 \mathrm{rpm}$ and baked at 200 ${ }^{\circ} \mathrm{C}$ for $1 \mathrm{~min}$ that result in an omnicoat thickness of $200 \mathrm{~nm}$ (Figure 2.c). This layer will act as a sacrificial layer to release microstructures after transferring step.

\subsection{Shadow-masking process}

The next step to be considered is the patterning of the structural layer on the top substrate. The present work proposes a versatile method to fabricate organic microcantilevers that does not require photomasking of materials as currently used [9] and thence, allows patterning of most organic materials, i.e., photo- or thermosensitive ones. For this, the proposed technique combines deposition and patterning in one step thanks to spray-coating through polymer microstencils.

\subsubsection{Organic microstencil fabrication}

Polymers are attractive materials to fabricate miniaturized microstencils for their low cost, good processability and flexibility [23-26]. Indeed, the polymer microstencil fabrication process proposed in this work requires only two photolithography steps. Also, flexible polymer microstencil can be adapted to several varieties of substrates such as flexible or non-flat ones. The process fabrication starts by the spincoating of a $200 \mathrm{~nm}$ omnicoat sacrificial layer on a silicon wafer to allow the release of microstencil from substrate after fabrication. The microstencil process results from the structuration of two levels of SU-8 epoxy-based negative photoresist (SU-8 ${ }^{\circledR} 3005$ and 3050) (Figure 3). SU-8 is a suitable material for this two steps device since several layers having different thicknesses $(0.5-200 \mu \mathrm{m})$ can be stacked-up by multiple spin-coating and UV-exposures to define high aspect ratio structures. In the present work, a first thin film of SU-8 was patterned to create apertures with high resolution. The influence of microstencil thickness was studied and chosen in order to obtain the most suitable PMMA thickness uniformity used as structural layer. For this, an $8 \mu \mathrm{m}$ thick SU-8 layer was spun, baked at $95^{\circ} \mathrm{C}$ for $3 \mathrm{~min}$ and UV-exposed with a dose of $150 \mathrm{~mJ} / \mathrm{cm}^{2}$ by using a manual mask aligner (MJB4, Suss Microtec. Corp, Germany). After, the layer was post-exposure baked at $65{ }^{\circ} \mathrm{C}$ for $1 \mathrm{~min}$ and 2 min at $95{ }^{\circ} \mathrm{C}$. But with this thickness, microstencil handling without cracking is challenging. Thus, circular apertures characterized by a diameter of $1 \mathrm{~mm}$ were patterned with a $100 \mu \mathrm{m}$ thickness of SU-8 that encircle the microstructure apertures on the thin layer, to strengthen the microstencil. This $100 \mu \mathrm{m}$ thick layer of SU-8 was successively spun and baked at $95{ }^{\circ} \mathrm{C}$ for $45 \mathrm{~min}$. Then, this layer was exposed with a dose of $250 \mathrm{~mJ} / \mathrm{cm}^{2}$ and baked at $65{ }^{\circ} \mathrm{C}$ for $1 \mathrm{~min}$ and $3 \mathrm{~min}$ at $95{ }^{\circ} \mathrm{C}$. After, both layers were simultaneously developed with propylene-glycol-monoether-acetate (PGMEA, Microchem Corporation). A low stress microstencil was required to allow a flat and conformal contact with the substrate during the spray-coating. In fact, a gap between the microstencil and the substrate would induce a loss of resolution in the geometries of the designed microstructures. Highly cross-linked SU-8 is known to induce residual stress in the patterned films. Several sources have been identified $[27,28]$ including residual solvent in the polymeric matrix [27], UV exposure dose [29], heating temperature for soft [28] and post-exposure bakes [27] and induced stress due to CTE mismatch between SU-8 and the substrate [30]. This can result in buckling of polymer microstencil and thus, gap increase during deposition. An efficient solution was proposed by introducing a hard-bake at the end of the SU-8 process, reducing residual stress gradient in highly cross-linked SU-8 films [27]. The hard-bake must be adapted to the thickness layer. For our process, a hard-bake step at 150 ${ }^{\circ} \mathrm{C}$ for $7 \mathrm{~min}$ was optimal. To finish the microstencil fabrication, the substrate was dipped in the omnicoat developer (MF 319) for $2 \mathrm{~h}$ to release the organic microstencil. 
Preprint - Journal of Micromechanics and Microengineering, vol. 21, 2011, 095021, 9 pages. (doi: 10.1088/0960-1317/21/9/095021).

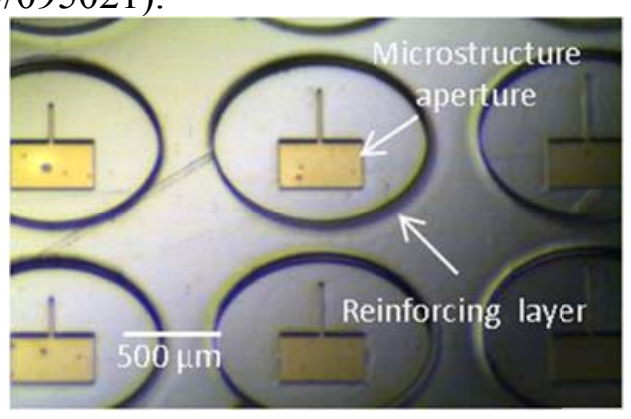

Figure 3. Optical image of SU-8 microstencil flattened on silicon substrate.

\subsection{2 spray-coating}

To pattern the microstructures that will be released, the next step of the shadow-masking process was the spray-coating through the polymer microstencil (Figure 2.d). First, the flexible shadow-mask was placed directly onto the substrate with a conformal contact. For this, electrostatic adhesion of shadow-mask was ensured by the use of a manual roll, allowing application of a slight pressure on top of the microstencil to improve adhesion. Then, there are several adjustable parameters involved in spray-coating that influence the thickness, resolution and uniformity of the resulting organic microstructures. Among others, the most important parameters are:

- The viscosity of the spray-coated solution that will have an effect on the droplet size.

- The spray pressure.

- The distance between the spray nozzle and the substrate.

- The duration of the spray.

- The aperture of the spray nozzle.

- The thickness of the stencil mask.

To achieve the patterning of organic microstructures, a commercial positive resist Polymethyl metacrylate $\left(\mathrm{NANO}^{\mathrm{TM}}\right.$ PMMA, 9\% solid content) from Microchem Corporation diluted in anisole from Sigma Aldrich was chosen as structural layer because this material does not require ne:cessarily photo-masking while properties of this material are well-known. To create a thin film of PMMA with the proposed method, a maximum distance from the spray nozzle was required to obtain a large spray-coated surface. In our case, a maximum distance of $12 \mathrm{~cm}$ has been used. A low spray pressure of $70 \mathrm{kPa}$ was applied in order to limit the spreading of solution on the microstencil edges. A short spray duration of 2 seconds was also required for each spray-coating step, to avoid apparition of polymer pool in the microstencil apertures. Instead, ten successive spray-coating steps were performed to fill the microstencil apertures. Following to spray-coating, microstencil was manually removed, and the patterned PMMA was thermally crosslinked at $180{ }^{\circ} \mathrm{C}$ for 1 min 30 seconds (Figure 2.e).

\subsection{Wafer-bonding process}

To obtain free-standing microcantilevers from a free structure, a manual, one-by-one transfer is widely employed, depending on the application reached $[15,16]$. For the wafer-level fabrication of organic MEMS, a wafer-bonding approach based on bonding of two SU-8 layers has been introduced [17, 18]. For this, SU-8 was used as adhesive and supporting layers for its high chemical and thermal stability and this resist was already shown as good candidate in bonding experiments, in the case of microfluidic chip fabrication [31]. However, optimization and quantification of such powerful process is necessary to define a generic fabrication protocol of organic free-hanging microstructures in a reproducible format. In this context, self-alignment pins are introduced during the parallel micromachining of both substrates, allowing the use of different substrates, not necessarily transparent ones. Also, polymerization degree of the partially reticulated SU-8 layers in contact is optimized to obtain strong bond after subsequent waferbonding combined with high fabrication yield.

\subsubsection{Photolithography process of the two SU-8 layers}

Two SU-8 layers, processed in parallel, are in contact during the wafer-bonding transfer: the transferring layer acting as adhesive layer and the layer supporting microstructures. For these layers, a SU-8 
Preprint - Journal of Micromechanics and Microengineering, vol. 21, 2011, 095021, 9 pages.

(doi: 10.1088/0960-1317/21/9/095021).

photolithography process was optimized to ensure their bonding during transfer. Among other parameters, several exposure doses were tested and studied before determining the optimal SU-8 photolithography process. Concretely, for the supporting layer, a $100 \mu \mathrm{m}$ thickness of SU-8 was spun on the top wafer and baked at $95{ }^{\circ} \mathrm{C}$ for $45 \mathrm{~min}$. Next, this layer was exposed with a dose of $190 \mathrm{~mJ} / \mathrm{cm}^{2}$ and baked at $65{ }^{\circ} \mathrm{C}$ for $1 \mathrm{~min}$ and $3 \mathrm{~min}$ at $95^{\circ} \mathrm{C}$ (Figure 2.g). The layer was then developed. In parallel, a $40 \mu \mathrm{m}$ thickness of SU-8 was spin-coated on the bottom wafer. The soft-bake was carried out at $95{ }^{\circ} \mathrm{C}$ for $18 \mathrm{~min}$. This SU-8 layer required an optimal UV-exposure dose of $160 \mathrm{~mJ} / \mathrm{cm}^{2}$. A post-exposure bake was successively performed at $65^{\circ} \mathrm{C}$ for $1 \mathrm{~min}$ and $4 \mathrm{~min}$ at $95^{\circ} \mathrm{C}$ before development (figure 2.f). Transferring chips characterized by a size of $25 \mathrm{~mm}^{2}$ were obtained with this layer.

\subsubsection{Wafers alignment: introduction of mechanical self-alignment pins}

Alignment of top and bottom wafers corresponds to the accurate placement of the supporting layer on the transferring chips. Currently, a Pyrex top wafer is used to allow alignment on the bottom wafer [17, 18, 31], restricting fabrication process to the use of at least one transparent wafer. In this work, we propose a mechanical self-alignment of wafers so that any specific properties for the substrates used are not required. The self-alignment was performed by incorporating alignment pin and jig structures to align the supporting layer with the transferring one. The pin area is $25 \mathrm{~mm}^{2}$ that can easily fit into jig structures. These alignment patterns ensure in plane self-alignment with an accuracy of $50 \mu \mathrm{m}$ that is enough for our process. Concerning alignment patterns fabrication, first, a $100 \mu \mathrm{m}$ thickness of SU-8 for pin structures was patterned by photolithography at the same time as the supporting layer (Figure 2.f). In parallel, an 80 $\mu \mathrm{m}$ thickness for jig structures was patterned onto the transferring layer (Figure 1h). With this method, the pin structures are mechanically clipped into the jig ones to ensure self-alignment between both wafers (Figure 2.i and Figure 4).

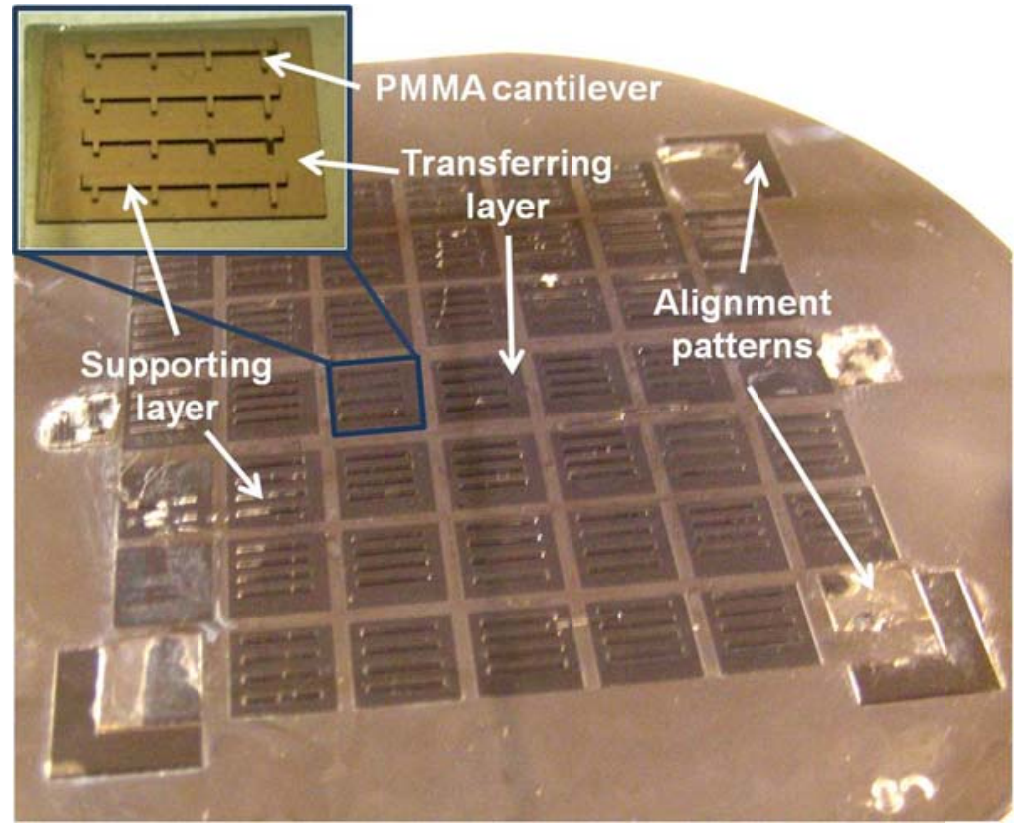

Figure 4. Optical image of transferred microstructures on 4-inches wafer. Inset shows a zoom view on a microcantilever chip (coated with a thin gold layer for observation) composed of four supports. Each support contains four PMMA cantilevers.

\subsubsection{Bonding process}

To allow the complete release of the free-standing microstructures, the aligned wafers were bonded together in order to transfer microstructures. Top and bottom wafers were compressed by applying a pressure and a temperature in order to bond the supporting layer on the transferring one (Figure 2.i). The transfer was realized by using a bonding device (Specac's Atlas Series Manual Hydraulic Press). A pressure of $300 \mathrm{kPa}$ was given as optimal value to bond two SU-8 layers as shown in previous work [31]. A temperature of $110^{\circ} \mathrm{C}$, that is above the glass transition temperature of the partially polymerized SU-8, was also recommended by [7] during $30 \mathrm{~min}$. In this case, both SU-8 layers became strongly bonded. 
Preprint - Journal of Micromechanics and Microengineering, vol. 21, 2011, 095021, 9 pages. (doi: 10.1088/0960-1317/21/9/095021).

\subsubsection{Sacrificial layer release}

To finish the fabrication process, the top wafer must be removed to obtain chips composed of polymer free-standing microstructures clamped on the bottom substrate obtained by collective fabrication. The omnicoat layer initially deposited was etched by using MF319 developer (Figure 2.j). The low gap between both wafers (about $150 \mu \mathrm{m}$ ) can prevent the release. Thus, to accelerate structures release, channels characterized by a width of $500 \mu \mathrm{m}$ were introduced on the transferring layer to enhance access to the sacrificial layer, reducing the release duration from $24 \mathrm{~h}$ to $6 \mathrm{~h}$. Last, the resulting wafer, containing free-standing microcantilevers, was cleaned according to a protocol where successive batches of omnicoat developer (to remove residual omnicoat), isopropanol, ethanol and deionized water were used. Cantilevers chips were then dried using a hotplate $\left(40{ }^{\circ} \mathrm{C}\right)$, ensuring rapid evaporation of water and thus, avoiding stiction of cantilevers onto the transferring layer.

\section{Results and discussion}

\subsection{Organic microcantilevers characterization}

By combining shadow-masking and wafer-bonding processing methods, we have successfully achieved the fabrication of free-standing PMMA cantilevers (Figure 4 and 5) characterized by a length ranging from 100 to $700 \mu \mathrm{m}$ and a width ranging from 50 to $250 \mu \mathrm{m}$. The geometries are in agreement with the design (maximum standard deviation of $2 \%$ for geometry with respect to designed mask), but the thickness profile is not uniform: the bottom surface of the cantilevers is not perfectly flat. In fact, protrusions at the microcantilever edges appear during the spray-coating through the shadow-masks (Figure 6). This effect has already been observed with other printing methods, such as screen-printing, nanoimprint or microfountain pen [32]. In our case, one origin of the phenomenon is capillary interactions that occur between the PMMA solution and SU-8 due to the wettability of anisole used as solvent for the PMMA solution [33]. To confirm this wettability effect, the SU-8 microstencil surface was made hydrophilic by submitting it to a short plasma oxygen event, resulting in the formation of PMMA clusters in the microstencil apertures after subsequent spray-coating, showing repulsive interactions between anisole and SU-8. At the moment, no concrete solution was found to avoid completely the edge effect, but the combined spray-coating/ shadow-masking process was optimized to obtain a convenient PMMA profile. For this, by using a mechanical profiler, the influence of the microstencil thickness and the solid content of the PMMA solution on the polymerized PMMA thickness profile has been studied (Figure 6.a). From this figure, it can be seen that at the center of the cantilever, the thickness is thin while at the edges it is increased, resulting in a thickness gradient with a parabolic profile. Also, the height of protrusions is defined by microstencil thickness: protrusions height increases with the microstencil thickness (Figure 6.a). A thin microstencil is thus required to limit this effect. But with a too thin SU-8 microstencil, there is not any PMMA material at the center of the structure and thus the whole microstructure surface cannot be covered. In this context, to restrict the protrusions height to a few micrometers, a microstencil characterized by a thickness of $8 \mu \mathrm{m}$ has been used while apertures have been then filled thanks to ten successive spray-coatings. For this, a homemade polymer solution that contains $4.5 \%$ of PMMA diluted in anisole has been used to reduce size of droplets. In this case, the thickness at the center of the cantilever is $5 \mu \mathrm{m}$, while at the edges it is $7.5 \mu \mathrm{m}$ (figure 6.b). With the optimized process, the aspect ratio between the height at the edge and the height at the center of the structures was clearly reduced, since an improvement from 8.3 to 1.5 was obtained. 
Preprint - Journal of Micromechanics and Microengineering, vol. 21, 2011, 095021, 9 pages. (doi: 10.1088/0960-1317/21/9/095021).

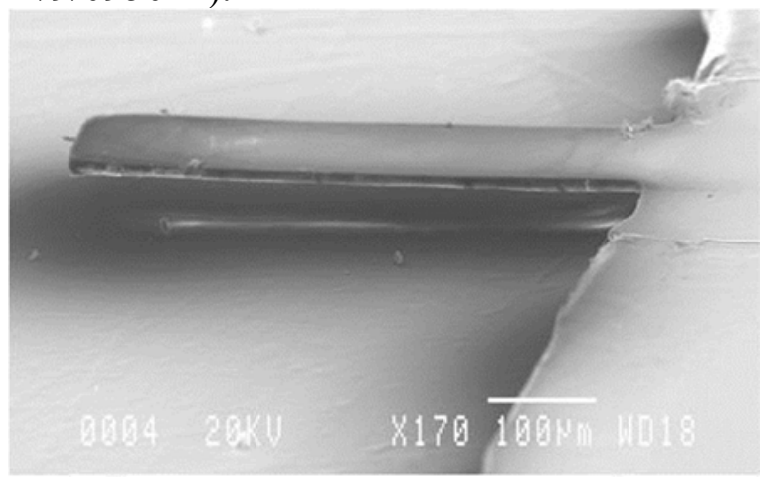

Figure 5. Scanning electron microscopy (SEM) image of PMMA microcantilever characterized by a thickness of $10 \mu \mathrm{m}$, a length of $520 \mu \mathrm{m}$ and a width of $100 \mu \mathrm{m}$.

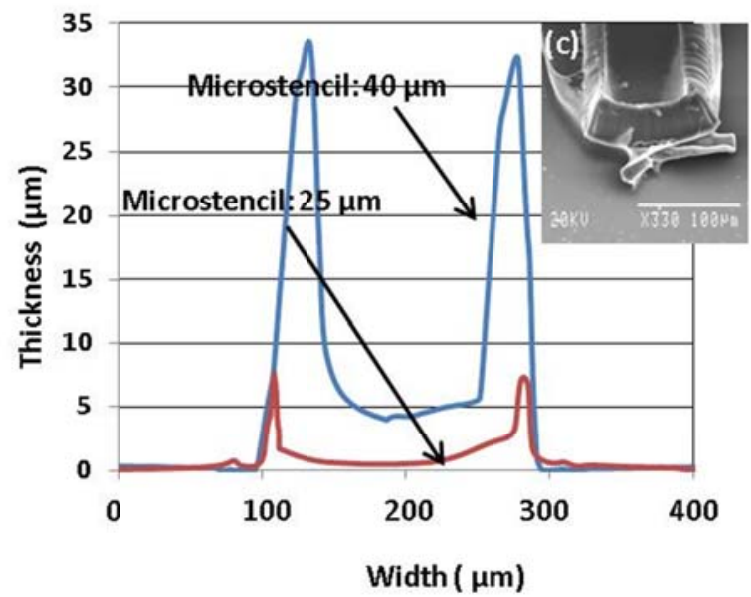

(a)

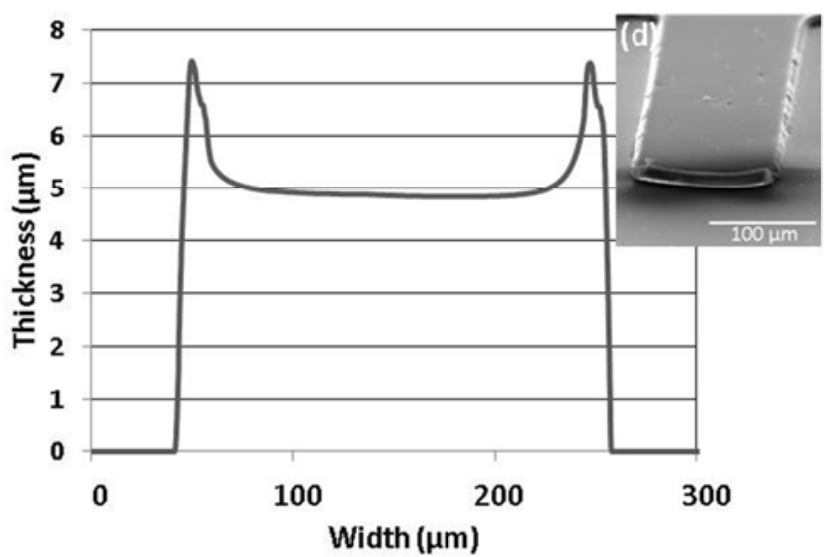

(b)

Figure 6. Profile measurement before transfer of PMMA cantilevers (a) Cantilever fabricated with one spray-coating step by using PMMA commercial solution and microstencils characterized by thicknesses of $25 \mu \mathrm{m}$ and $40 \mu \mathrm{m}$. (b) Cantilever fabricated with optimal spray-coating parameters and by using microstencil characterized by a thickness of $8 \mu \mathrm{m}$. (c) Scanning electron microscopy (SEM) image of PMMA microcantilever fabricated with a microstencil characterized by a thickness of $40 \mu \mathrm{m}$. (d) Scanning electron microscopy (SEM) image of PMMA microcantilever fabricated with optimal spraycoating parameters by using microstencil characterized by a thickness of $8 \mu \mathrm{m}$.

\subsection{Bonding process characterization}

Using the wafer-bonding method, we have achieved the large scale fabrication of clamped free-standing organic cantilevers on 4-inches wafers. The transferring technique is suitable for integration in industrial processes since a fabrication yield of $100 \%$ was obtained (figure 4 ).

With this method, the transfer requires a SU-8 crosslinking surface reaction between the supporting and the transferring layers to ensure their bonding. This reaction results from association of three parameters: the pressure that ensures contact between layers, the temperature and the SU-8 crosslinking level that allows a crosslinking reaction between both surfaces in contact.

Temperature and pressure have already been investigated in previous works for the fabrication of microfluidic chips [34, 31]. Obtained results recommend a pressure of $300 \mathrm{kPa}$ to obtain optimal bonded area. A bonding temperature of $110^{\circ} \mathrm{C}$ is generally used [7] that is compatible with PMMA. However, a lower temperature could be interesting if the structural material is not resistant at $110{ }^{\circ} \mathrm{C}$ [34]. Moreover, the SU-8 crosslinking level, resulting mainly from UV-exposure dose, is also a critical parameter in the bonding process. Thence, it is particularly important to investigate the influence of the UV-exposure dose on the transfer quality. For this, the exposure dose was varied around the value recommended by SU-8 datasheet. Figure 7 shows the bonded area and the bond strength for several UV-exposure doses applied 
Preprint - Journal of Micromechanics and Microengineering, vol. 21, 2011, 095021, 9 pages. (doi: 10.1088/0960-1317/21/9/095021).

to the transferring layer and the supporting one. The UV-exposure dose is defined by thickness of the layer. UV-exposure doses were thus chosen in order to have the same crosslinking level for both layers in contact. From figure 7, it can be seen that the bond area decreases with dose increment. This indicates that a high yield was associated to a low crosslinking level of SU-8 layers before transfer that allows complete SU-8 thermal polymerization during the transfer. Also, the bond strength has been investigated by using a Dage 2000 shear station. Results confirm that the bond strength is high for a low exposure dose. A strength value around $10 \mathrm{MPa}$ is recommended to ensure strong bond between two SU-8 layers [31]. In this case, the exposure dose for the transferring layer must be below $240 \mathrm{~mJ} / \mathrm{cm}^{2}$ and the one for the supporting layer below $270 \mathrm{~mJ} / \mathrm{cm}^{2}$. However, to obtain a maximum bonded area of $100 \%$ and a strong bond, a dose of $160 \mathrm{~mJ} / \mathrm{cm}^{2}$ for the transferring layer and $190 \mathrm{~mJ} / \mathrm{cm}^{2}$ for the supporting one were found to be optimal, since it corresponds to a bond strength of $50 \mathrm{MPa}$.

Using layers with low crosslinking levels during the bonding process, a reflow of the SU-8 layers can occur that damages the patterned layers. In fact, a decrease of crosslinking level causes a loss of SU-8 robustness, inducing a possible damage of the structures under the pressure and temperature used for bonding. Figure 8.a shows the spreading of SU-8 transferring layer and the flattening of the supporting layer due to too low UV-exposure doses that are respectively 80 and $110 \mathrm{~mJ} / \mathrm{cm}^{2}$. On the contrary, with optimal exposure doses, a clean and strong bond occurs between the two SU-8 layers (figure 8.b).

To summarize, to improve the wafer-bonding process based on SU-8, with a bonded area of $100 \%$, a strong bond and a limited reflow, the optimal UV-exposure doses must be $160 \mathrm{~mJ} / \mathrm{cm}^{2}$ and $190 \mathrm{~mJ} / \mathrm{cm}^{2}$ for respectively the transferring and the supporting layers. With these values, the overly level of polymerized layers is avoided before transfer that ensures a strong adhesion of bonded layers during the transfer. But SU-8 layers are nevertheless polymerized enough to prevent their damage (Figure 8.b). The transfer process has been thus optimized to standardize large-scale microfabrication of organic microcantilevers.

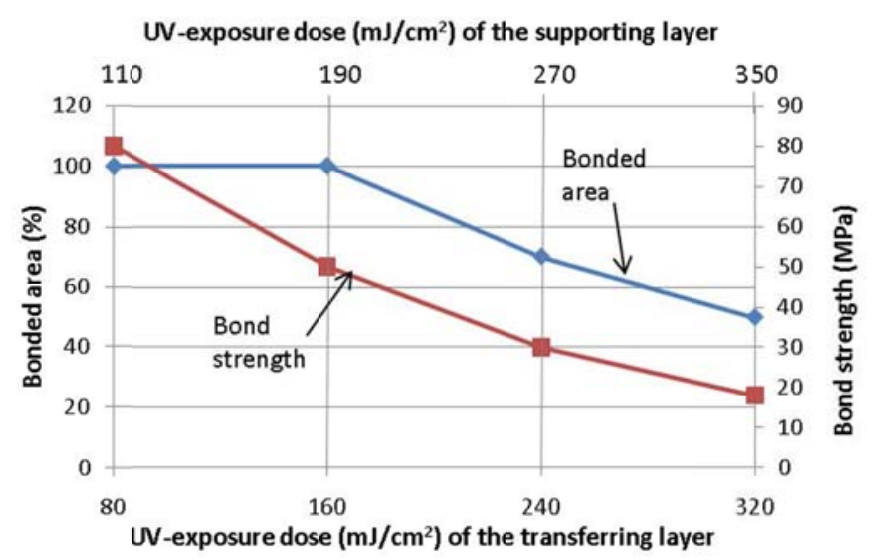

Figure 7. Bonded area and bond strength versus UV exposure doses of SU-8 supporting and transferring layer.

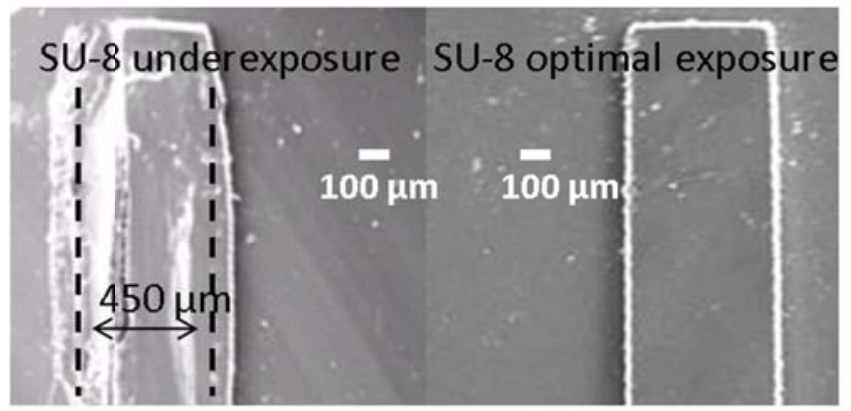

(a)

(b)

Figure 8. Scanning electron microscopy (SEM) image of SU-8 transferring and transferred supporting 
Preprint - Journal of Micromechanics and Microengineering, vol. 21, 2011, 095021, 9 pages. (doi: 10.1088/0960-1317/21/9/095021).

layers for respectively (a) UV-exposure doses of 80 and $110 \mathrm{~mJ} / \mathrm{cm}^{2}$ and (b) 160 and $190 \mathrm{~mJ} / \mathrm{cm}^{2}$.

\subsection{Preliminary mechanical characterization.}

To confirm the potential of such organic microcantilevers as flexible devices, the next step concerned the measurement of mechanical properties of the patterned structural layer. For this, out-of-plane flexural bending resonant modes were studied using a laser Doppler vibrometer (Polytec MSA500); Young's modulus of the structured layer was then calculated thanks to equation (1) [35]:

In this equation, $E$ represents the cantilever Young's modulus, $f_{n}$ is the resonance frequency of the $n^{\text {th }}$ mode, $\mathrm{L}$ and $\mathrm{h}$ are respectively the length and thickness of the microcantilever, $\rho$ represents the mass density of the material, and $\lambda_{n}$ the eigenvalue of the $n^{\text {th }}$ resonance mode. For PMMA cantilevers characterized by a length of $200 \mu \mathrm{m}$ and an average thickness of $16 \mu \mathrm{m}$, a resonance frequency of 122.8 $\mathrm{kHz}$ was measured for the first flexural mode, giving a Young's modulus value of $3.7 \pm 0.5 \mathrm{GPa}$. Also, to demonstrate the versatility of the transferring process, SU-8 cantilevers (Figure 9) were fabricated using standard photolithography. A resonance frequency of $10.7 \mathrm{kHz}$ was obtained for a SU-8 cantilever characterized by a length of $490 \mu \mathrm{m}$ and a thickness of $8 \mu \mathrm{m}$, giving a Young's modulus of $4.0 \pm 0.5 \mathrm{GPa}$. A similar method was already proposed for the determination of Young's modulus of SU-8 cantilevers [10]. Values obtained ranged from 3.5 GPa and 4.5 GPa, depending on the thickness of SU-8. Values obtained in present work are in agreement with those previously described [10] and those obtained in other works [27, 36], validating the method for both PMMA and SU-8 materials. Also, the low values obtained show a great potential for these organic free-standing microcantilevers to obtain large mechanical deflections for a variety of applications, including chemical sensing in static mode.

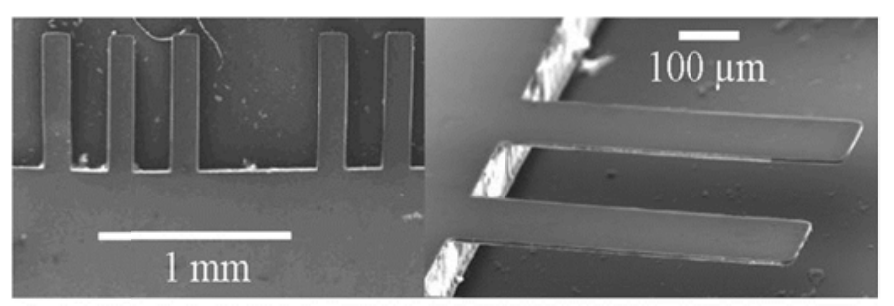

(a)

(b)

Figure 9. Scanning electron microscopy (SEM) image of SU-8 microcantilevers. (a) General view of transferred SU-8 microcantilevers. (b) Zoom view of SU-8 microcantilevers characterized by a thickness of $8 \mu \mathrm{m}$, a length of $640 \mu \mathrm{m}$ and a width of $140 \mu \mathrm{m}$.

\section{Conclusion}

A versatile all-organic processing method for the large scale fabrication of free-standing polymer microstructures was proposed. This method results from the association of two different approaches: shadow-masking and wafer-bonding. The first approach is coherent for the patterning of thermo and photosensitive polymer materials. This method consists in the spray-coating of the structural layer through a flexible microstencil. Thereby, deposition and patterning were performed in one step. Moreover, introduction of polymers in microstencil fabrication makes this process simple at low-cost. The second approach is particularly adapted for industrial integration of open microstructure fabrication such as microcantilevers, bridges, or microchannels. Indeed, the process transfer has been optimized in order to have a fabrication yield of $100 \%$ with a bond strength of $50 \mathrm{MPa}$. Also, this method is compatible with several patterning methods such as photolithography and nanoimprinting. By combining both approaches, PMMA microcantilevers have been successfully obtained. Then, to confirm the versatility of the waferbonding approach, SU-8 microcantilevers were fabricated using standard photolithography. Preliminary mechanical characterization of resulting PMMA and SU-8 cantilevers are in agreement with the literature 
Preprint - Journal of Micromechanics and Microengineering, vol. 21, 2011, 095021, 9 pages. (doi: 10.1088/0960-1317/21/9/095021).

values. These organic cantilevers, characterized by a low Young's modulus, have the potential to serve as highly sensitive transducing devices in biological and chemical sensing applications. Indeed, for a $200 \mu \mathrm{m}$ long bi-layered cantilever, the static deflection due to swelling effects in an organic sensitive layer will be thirty times higher when using these organic materials instead of silicon (theoretical calculation using a modified Stoney equation [37]). Based on this approach, work is now in progress for the development of tuned Young's modulus organic materials for highly sensitive biological and chemical sensing applications in static mode.

\section{References}

[1] Burg T P, Mirza A R, Milovic N, Tsau C H, Popescu G A, Foster J S and Manalis S R 2002, J. Microelectromech. Syst. 15 1466-1476

[2] Burg T P, Godin M, Knudsen S M, Shen W, Carlson G, Foster J S, Babcock K, and Manalis S R 2007 Nature 446 1066-1069

[3] Hwang K S, Lee S M, Eom K, Lee J H, Lee Y S, Park J H, Yoon D S, and Kim T S 2007 Biosensors and Bioelectronics 23 459-465

[4] Kim S J, Onob T and Esashi M, 2007 J. Appl. Phys. 102 104-304

[5] Van Oosten C L, Cees W. Bastiaansen M and Broer J D 2009 Nature Materials 8 677- 682

[6] Matyjaszewski K 2005 Prog. Polym. Sci. 30858

[7] Agirregabiria M, Blanco F J, Berganzo J, Arroyo M T, Fullaondo A, Mayoraa K and Ruano-Lopez J M 2005 Lab on a chip 5 545-552

[8] Seena V, Rajorya A, Pan P, Mukherji S, Ramgopal Rao V, 2009, Solid State Sciences 11 1606-1611

[9] Abgrall P, Conedera V, Camon H, Gue A M and Nguyen N T 2007 Electrophoresis 28 4539-4551

[10] Ezkerra A, Fernandez L J, Mayora K and Ruano-Lopez J M 2007 J. Micromech. Microeng. 17 22642271

[11] Bhusari D, Reed H. A, Wedlake M, Padovani A M and al. 2001 J. Microelectromech. Syst. 10 400408

[12] Oh K W and Ahn C H 2006 J. Micromech. Microeng. 16 R13-39

[13] Chung C and Allen M 2005 J. Micromech. Microeng. 15 N1-N5

[14] Dubourg G, Fadel-Taris L, Dufour I, Pellet C and Ayela C 2010 Proceedings of the MNE conference (Micro and Nano Engineering) Ginoa, Italy

[15] Johansson A, Blagoi G and Boisen A 2006 Appl. Phys. Lett. 89173505

[16] Greve A, Keller S, Vig A L, Kristensen A, Larsson D, Yvind K, Hvam J M, Cerruti M, Majumdar A and Boisen A 2010 J. Micromech. Microeng. 20009015

[17] Nordström M, Calleja M, Hübner J and Boisen A, 2008 J. Micromech. Microeng. 18015017

[18] Murillo G, Davis Z J, Keller S, Abadal G, Agusti J, Cagliani A, Noeth N, Boisen A and Barniol N 2010 Microelec. Eng. 87 1173-1176 
Preprint - Journal of Micromechanics and Microengineering, vol. 21, 2011, 095021, 9 pages.

(doi: 10.1088/0960-1317/21/9/095021).

[19] Guillon S, Saya D, Mazenq L, Perisanu S, Vincent P, Lazarus A, Thomas O and Nicu L 2011 Nanotechnology 22245501

[20] Fadel-Taris L, Ayela C, Josse F, Heinrich S, Brand O, Saya D and Dufour I 2011, Proceedings of IEEE International Frequency Control Symposium San Francisco, USA

[21] Selvarasah S, Chao S H, Chen C L, Sridhar S , Busnaina A, Khademhosseini A and Dokmeci M R 2008 Sensors and Actuators A 145-146 306-315

[22] Sidler K, Cvetkovic N V, Savu V, Tsamados D, Ionescu A M and Brugger J 2009 Procedia chemistry 1762

[23] Kim G, Kim B and Brugger J 2003 Sensors and Actuators A 107 132-136

[24] Choi J H and Kim G M 2011 International J. Precision engineer. And Manufacturing 12 165-168

[25] Atsuta K, Suzuki H and Takeuchi S 2004 Proceedings of the $8^{\text {th }}$ International Conference on Miniaturized Systems for Chemistry and Life Sciences, Malmo, Sweden

[26] Pal R, Sung K E and Burns M A 2006 Langmuir 22, 5391-5397

[27] Keller S, Haefliger D and Boisen A 2010 J. Micromech. Microeng. 20045024

[28] Anhoj T A, Jorgensen A M, Zauner D A and Hubner J 2006 J. Micromech. Microeng. 161819

[29] Lee K Y, LaBiance N, Rishton S A, Zolgharnain S, Gelorme J D, Shaw J and Chang T H P 1995, J. Vac. Sci. Technol. B 133012

[30] Keller S, Blagoi G, Lillemose M, Haelfiger D and Boisen A 2008 J. Micromech. Microeng. 18 125020

[31] Blanco F J, Agirregabiria M, Garcia J, Berganzo J, Tijero M, Arroyo M T, Ruano J M, Aramburu I and Mayora K 2004 J. Micromech. Microeng. 14 1047-1056.

[32] Gilles S, Matthias Meier M, Prömpers M, Van der Hart A, Kügeler C, Offenhäusser A and Mayer D 2009 Microelectronic Engineering 86 661-664

[33] Low H L, Dumond J, Chong K, Yamamoto K and Amamiya S 2010 United States Patent Application Publication US2010/0291723 A1

[34] Pang C, Zhao Z, Du L and Fang Z 2008 Sensors and Actuators A 147 672-676

[35] Blevins R D 1979 Robert E Kieger Publihing Compagny

[36] Ferrell N and Hansford D 2005 Journal of vacuum science and technology 23 811-819

[37] Wenzel M J, Josse F, Heinrich S M, Yaz E and Datskos P G 2008 J. Appl. Phys. 103, 064913 